\title{
Current Sensor Fault Estimation in the $(d, q)$ rotating synchronous frame
}

\author{
D. Diallo, Senior Member, IEEE, S. Diao \\ GeePs $\mid$ Group of electrical engineering - Paris \\ UMR CNRS 8507, CentraleSupélec, Univ. Paris-Sud, Université Paris- \\ Saclay, \\ Sorbonne Universités, UPMC Univ Paris 06 \\ 11 rue Joliot-Curie, Plateau de Moulon 91192 Gif-sur-Yvette, France \\ Demba.diallo@geeps.centralesupelec.fr
}

\begin{abstract}
In this paper, a current sensor fault estimation using the transformed currents in the Park synchronous rotating frame is proposed. We show that from an analytical model, the fault characteristics can be retrieved. From experimental raw data collected from a Permanent Magnet Synchronous Machine drive, the gain or offset fault characteristics (amplitude and frequency) have been estimated with an average error of $10 \%$. For incipient fault, despite the analytical model, the estimation might be tedious because the fault is concealed by the noise. In this case the Kullback-Leibler Divergence between two Probability Density Functions can be computed and the fault estimated from the value of the divergence. If the distributions are Gaussian, the closed form of the divergence allows the fault estimation. However even if the data are not perfectly Gaussian-distributed, the closed form can still be used despite an overestimation of the fault characteristics that is preferable as it is a safety margin.
\end{abstract}

Keywords: Current sensor fault, Fault Estimation, Park transform, Kullback-Leibler Divergence

\section{I- INTRODUCTION}

In every process, data are measured through sensors. In AC drives, the most usual sensors are current sensors, position or speed sensor, temperature sensor, voltage sensor, torque transducer and accelerometers.

Like other components, sensors can be affected by faults such as gain fault, offset or bias fault and noise. When the measured variables are used in closed loops, the controllers can be tuned to be robust against these perturbations. However when the fault becomes significant, the outputs of the controllers saturate and lead to a degradation of the performances. For aerospace applications, a solution is to choose hardware redundancy as a conservative design method despite the additional. Otherwise a Fault Tolerant Controller is engaged with the use of virtual sensors (software redundancy) that estimate the missing or bad information from the remaining relevant information [1-3].

To detect and isolate current sensor fault, different methods are proposed in the literature. They can be classified in two families:

- Observer-based methods [4-7],

\author{
C. Delpha, Member, IEEE \\ L2S | Laboratoire des Signaux et Systèmes \\ UMR CNRS 8506, CentraleSupélec, Univ. Paris-Sud, Université Paris- \\ Saclay, \\ 3 rue Joliot-Curie, Plateau de Moulon 91192 Gif-sur-Yvette, France \\ claude.delpha@12s.centralesupelec.fr
}

- Pattern recognition after transformation of the currents in the Park synchronous rotating frame or in the Concordia stationary reference frame. [8].

The methods based on observer (deterministic or stochastic) estimate the currents from a model and compute residuals that are compared to a constant or variable threshold. But the fault amplitude itself is not estimated. In fact they are usually associated to a Fault Tolerant Control, which ensures the continuity of operation.

The methods based on pattern recognition analyse the properties of the current trajectory in a reference frame. The distortions from the healthy pattern are computed and analysed to generate alarms.

In both methods, the main goal is to extract the characteristics of the features used for fault detection and isolation. The fault characteristics are not determined. And yet this information may be important for maintenance purposes or even for control issues.

This paper proposes a method to determine the current sensor fault characteristics.

The method is based on the analysis of the probability density functions of the currents transformed in the Park rotating synchronous frame.

\section{II- FAULt MODELLING}

To estimate the fault characteristics, a model should be available with the fault characteristics as parameters. This model can be data-driven or analytical and is derived from the features used to detect and isolate the fault. In the first approach, signal processing, identification and optimisation algorithms are used to extract these parameters [9]. In the second approach as displayed by the flowchart in Figure 1, an analytical model is developed and the parameters are extracted.

Three types of fault model are generally presented in the time domain corresponding to their occurrence: abrupt 
fault, intermittent fault and gradual fault. This is illustrated in Figure 2.

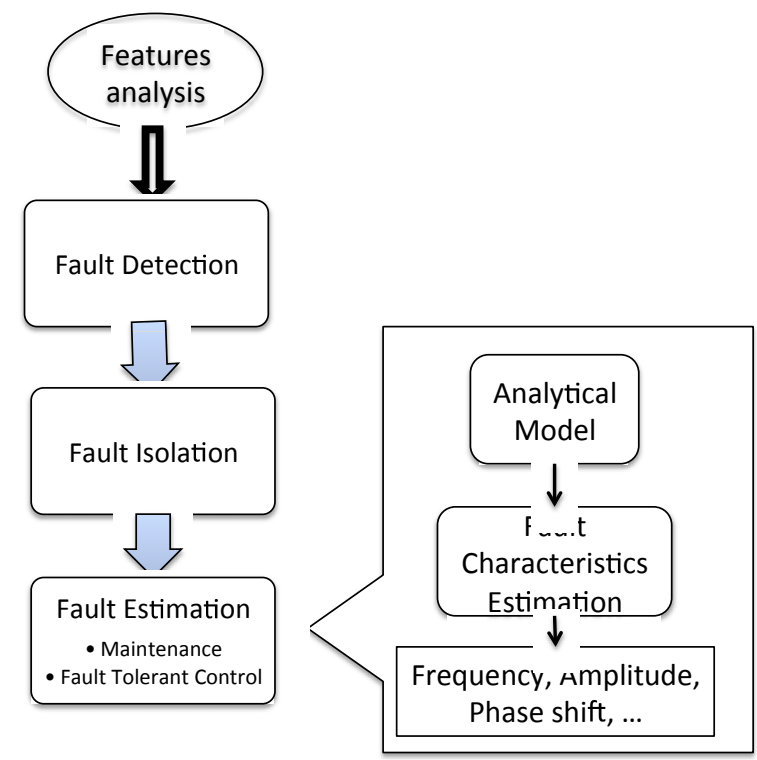

Figure 1. Fault Detection, Isolation and Estimation processs

However this representation is relevant to explain the fault type but for the fault estimation, the model should be based on the features used for fault detection. In [10-11] an analytical model of the Kullback-Leibler Divergence has been derived and the fault severity has been estimated from the divergence value $\hat{a}=f^{-1}(K \hat{L} D)$.
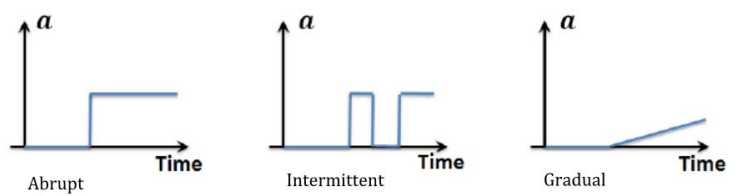

Figure 2. Fault types

\section{CUrRent SEnsor Fault Modelling}

The most usual current sensor type used in AC drives is the active Hall Effect Sensor. From the actual current flowing in the machine winding to the current used in the digital control, the measured variable can be affected by different faults. The information can be lost (power outage of the sensor) degraded with an offset, a gain error or corrupted by significant noise. Figure 3 shows an overview of this process where ADC is the Analog-todigital Converter.

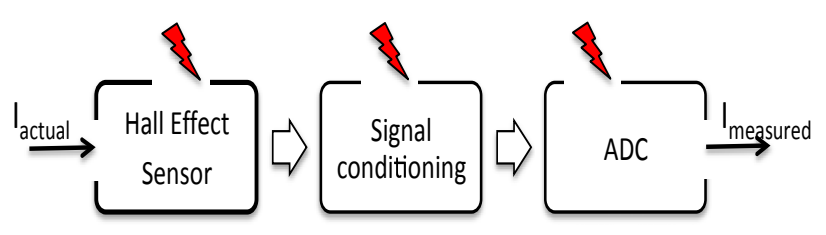

Figure 3. Current sensing flowchart

As a consequence, the current sensor fault can be modelled as the relation between the actual and the measured current with the following relation:
$I_{\text {measured }}=(1+G) I_{\text {actual }}+\delta I_{\text {actual }}+\varepsilon$

where $\mathrm{G}$ is the gain error, $\delta I_{\text {actual }}$ is the offset and $\varepsilon$ is the noise.

In the natural $(a, b, c)$ frame, if sinusoidal output currents are assumed, they can be expressed as :

$i_{a}=I \sin (\theta)$

$i_{b}=I \sin (\theta-2 \pi / 3)$

$i_{c}=I \sin (\theta-4 \pi / 3)$

Following the Park transform, the currents $\left(i_{d}, i_{q}, i_{0}\right)$ in healthy conditions are DC variables:

$i_{d h}=0$

$i_{q h}=\sqrt{\frac{3}{2}} I$

$i_{0 h}=0$

In the following, the homopolar component $i_{0}$ will no longer be considered even it may contain relevant information. Only the $\mathrm{d}$ and q currents will be studied.

In case of gain fault $(G \neq 0)$, the transformed currents become:

$$
\begin{aligned}
& i_{d f}=i_{d h}+\frac{1}{\sqrt{6}} G I \sin (2 v)+\zeta \\
& i_{q f}=i_{q h}-\frac{1}{\sqrt{6}} G I(1+\cos (2 v))+\zeta
\end{aligned}
$$

In case of offset fault, the transformed currents are:

$$
\begin{aligned}
& i_{d f}=i_{d h}+\sqrt{\frac{2}{3}} \delta I \cos (v)+\zeta \\
& i_{q f}=i_{q h}-\sqrt{\frac{2}{3}} \delta I \sin (v)+\zeta
\end{aligned}
$$

where $\zeta$ is the noise and $v=\theta, \theta-\pi / 3, \theta-2 \pi / 3$ depending of the affected phase angle.

As it can be noticed in (4) and (5), if the d and q currents are used as features for fault detection and diagnosis, the analytical model contains all the parameters related to the fault characteristics.

\section{CURRENT SENSOR FAULT ESTIMATION}

In this section, from experimental results, the sensor fault will be analysed and the fault estimated thanks to (4) and (5).

The tests are run on a $1.1 \mathrm{~kW}$ salient PMSM with a nominal torque of $3 \mathrm{Nm}$ and $3000 \mathrm{rpm}$ for the nominal speed. The other PMSM parameters are given in Table I. The switching frequency is $20 \mathrm{kHz}$. A powder brake is used to provide the load. The sampling time is $\mathrm{T}_{\mathrm{e}}=0.1 \mathrm{~ms}$.

TABLE I

MACHINE PARAMETERS

\begin{tabular}{ccc}
\hline \hline \multirow{2}{*}{ Symbol } & Quantity & Value and Unit \\
\hline \multirow{2}{*}{$\Phi_{m}$} & Magnetic flux & $0.153 \mathrm{~Wb}$ \\
$L_{d}$ & D axis inductance & $3.5 \mathrm{mH}$ \\
$L_{q}$ & Q axis inductance & $4.5 \mathrm{mH}$
\end{tabular}




$\begin{array}{ccc}R_{s} & \text { Resistance } & 1.66 \Omega \\ J & \text { Inertia } & 6^{*} 10^{-3} \mathrm{~kg} / \mathrm{m}^{2} \\ f & \text { Viscous friction } & 0.5^{*} 10^{-3} \mathrm{Nm} / \mathrm{s} \\ p & \text { Pole pairs } & 3 \\ V_{n} & \text { Nominal voltage } & 200 \mathrm{~V} \\ I_{n} & \text { Nominal current } & 6 \mathrm{~A}\end{array}$

A. Offset fault

In the phase B measured current, an offset of $1 \mathrm{~A}$ is introduced between 2.8 and $6.5 \mathrm{~s}$. The fault amplitude corresponds to $16 \%$ of the healthy current at steady state. The residuals $\begin{gathered}i_{d f}-i_{d h} \\ i_{q f}-i_{q h}\end{gathered}$ are plotted in figure 4. We can observe an additional sinusoidal component. These results confirm the theoretical results forecast from (5).

The amplitude of this additional component is proportional to the offset fault.

From the estimated amplitudes of this additional component in both components, we have

$$
\delta \hat{I}_{d}=0.98 A
$$$$
\delta \hat{I}_{q}=1.2 \mathrm{~A}
$$

The average value of the estimated offset is $\delta \hat{I}=1.1 A$

The other fault characteristic is the frequency of the additional sinusoidal component estimated at $12.7 \mathrm{~Hz}$ corresponding to the actual electrical frequency (190 rpm). In absence of faults, $\delta \hat{I}_{d q}$ is not null and a threshold must be set.

If the threshold is too high, the number of misdetection will increase and if it is too low, the number of false alarm will increase. Therefore, the tuning of the optimal threshold is a compromise.
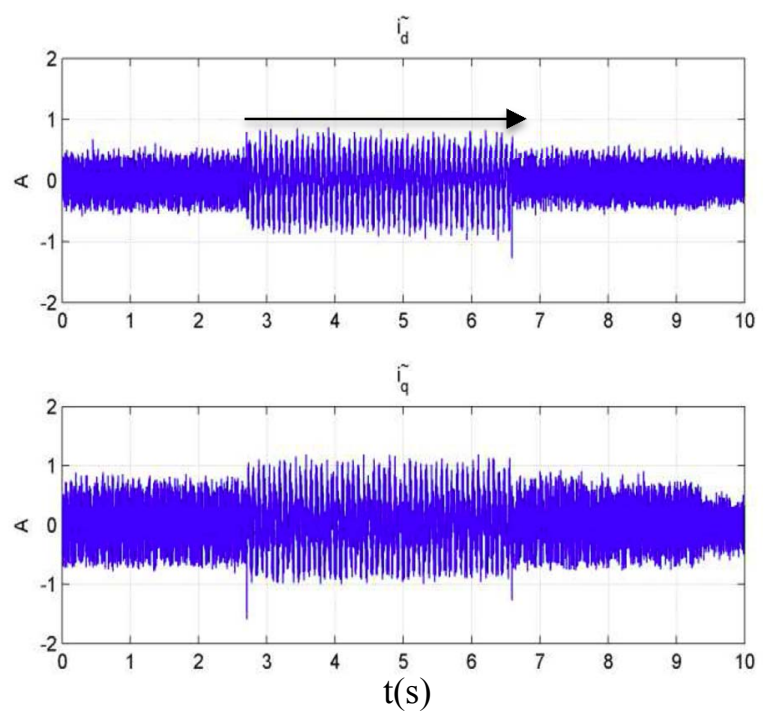

Figure 4. Transformed currents in case of offset fault

B. Gain fault

On the phase A measured current ( $\mathrm{I}=1.5 \mathrm{~A})$, a gain error $\mathrm{G}$ $=0.6$ is introduced between 2.3 and 6.s. The residuals $i_{d f}-i_{d h}$
$i_{q f}-i_{q h}$ are plotted in figure 5 . We can observe an additional sinusoidal component. These results confirm the theoretical results forecast from (4). The amplitude of this additional component is proportional to the offset fault.

From the estimated amplitudes of this additional component in the $q$ component, we have $\frac{\hat{G}_{q} I}{\sqrt{6}}=0.4 \mathrm{~A}$

The estimated gain is therefore $\hat{G}_{q}=0.65$.

The other fault characteristic if the frequency of the additional sinusoidal component estimated at $79.6 \mathrm{~Hz}$ corresponding to twice the actual electrical frequency $(600$ rpm).
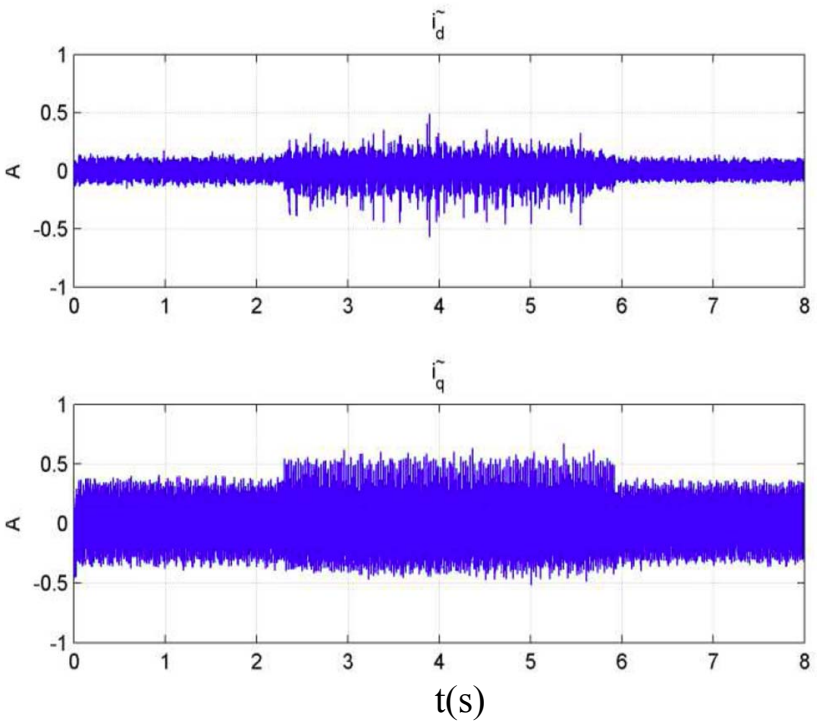

Figure 5. Transformed currents in case of gain fault

For both fault types, thanks to the analytical model, we have estimated the fault characteristics (severity or amplitude and frequency) from raw data with less than $10 \%$ error. This can be improved with a better processing of the data.

However when the fault becomes incipient, meaning that the Signal to Noise Ratio (SNR) is high and the Fault to Noise Ratio (FNR) is almost null or negative, the additional components in (4) and (5) might be hidden by the noise. As an example, in figure 6 , with $\mathrm{SNR}=30 \mathrm{~dB}$ and an offset fault of $0.01 \mathrm{~A}$ corresponding to an FNR of $16.5 \mathrm{~dB}$, the transformed current $i_{q}$ is plotted in healthy and faulty condition. 


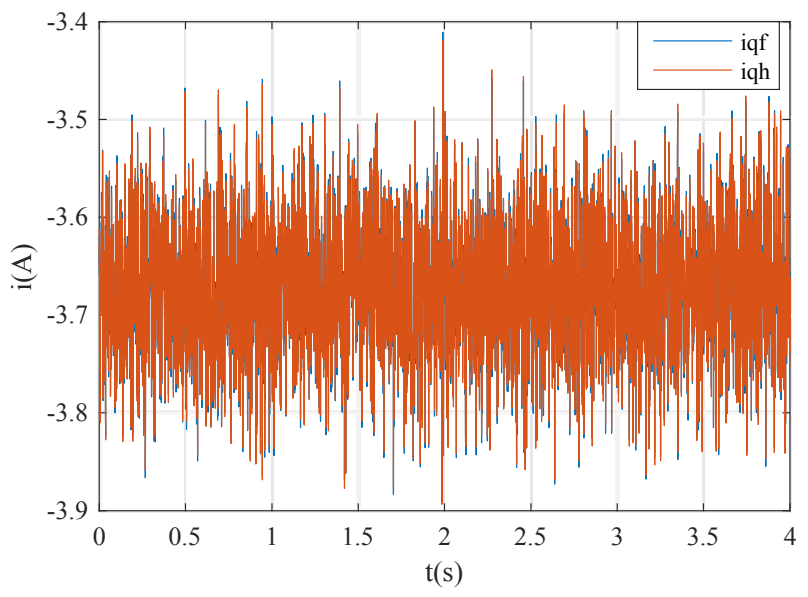

Figure 6. q axis transformed currents in case of offset fault $(\mathrm{SNR}=30 \mathrm{~dB}, \mathrm{FNR}=-16.5 \mathrm{~dB})$

In this case statistical indicators are more suitable for fault detection, isolation and estimation [12].

It has been shown in [11] that the Kullback-Leibler Divergence is very efficient for detecting incipient faults by computing the slight dissimilarities between two pdf as illustrated in figure 7 .

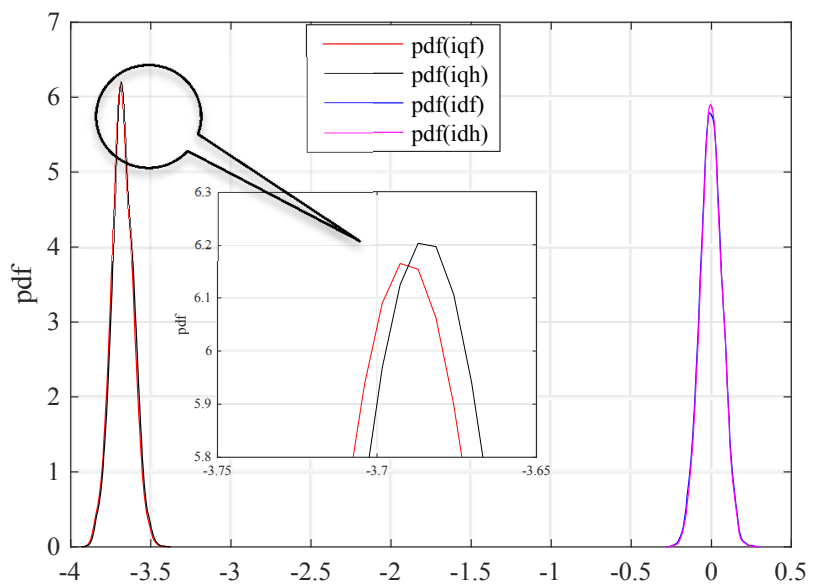

Figure 7. Pdf of the currents in the Park transformed frame $(\mathrm{SNR}=30 \mathrm{~dB}, \mathrm{FNR}=-16.5 \mathrm{~dB})$

\section{Probability DENSITY Functions}

\section{The Kullback-Leibler Divergence}

For discrimination between two continuous probability distribution functions (pdfs) $f(x)$ and $g(x)$ of a random variable $\mathrm{x}$, the Kullback-Leibler Information is defined as:

$$
I(f / / g)=\int f(x) \log \frac{f(x)}{g(x)} d x
$$

The divergence is the symmetric version of the Information and is given by:

$$
K L D(f, g)=I(f / / g)+I(g / / f)
$$

For normal densities $f$ and $g$ such that

$$
\begin{aligned}
& f \approx N\left(\mu_{1}, \sigma_{1}^{2}\right) \\
& g \approx N\left(\mu_{2}, \sigma_{2}^{2}\right)
\end{aligned}
$$

where $\mu_{1}, \mu_{2}$ are the means and $\sigma_{1}^{2}, \sigma_{2}^{2}$ are the variances for $f$ and $g$ respectively, (7) has an analytical form given by:

$$
K L D(f, g)=\frac{1}{2}\left[\frac{\sigma_{2}^{2}}{\sigma_{1}^{2}}+\frac{\sigma_{1}^{2}}{\sigma_{2}^{2}}+\left(\mu_{1}-\mu_{2}\right)^{2}\left(\frac{1}{\sigma_{1}^{2}}+\frac{1}{\sigma_{2}^{2}}\right)-2\right]
$$

The probability density functions (pdf) of the phase currents $\left(i_{a}, i_{b}\right.$ and $\left.i_{c}\right)$ are plotted in Figure 8.

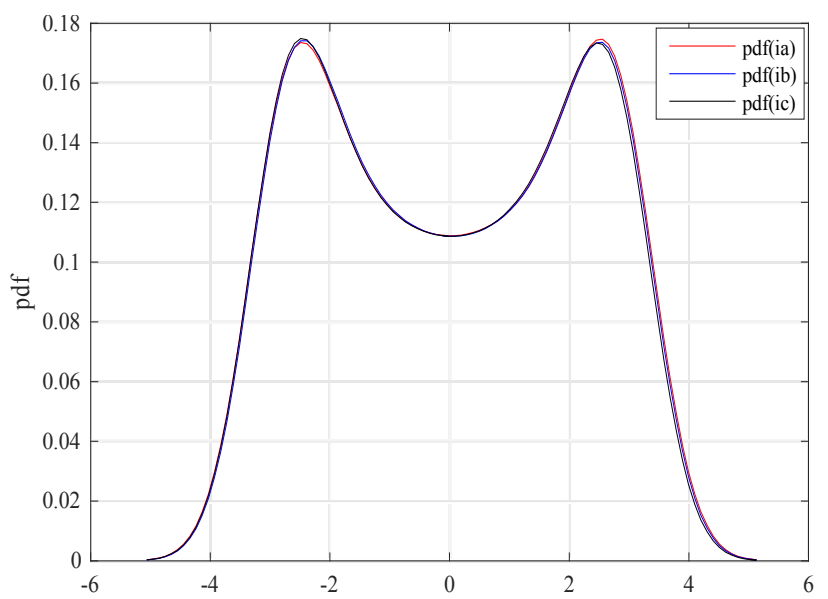

Figure 8. Pdf of the currents in the natural frame $(\mathrm{SNR}=30 \mathrm{~dB}, \mathrm{FNR}=-16.5 \mathrm{~dB})$

Figure 9 represents the pdfs of the transformed currents.

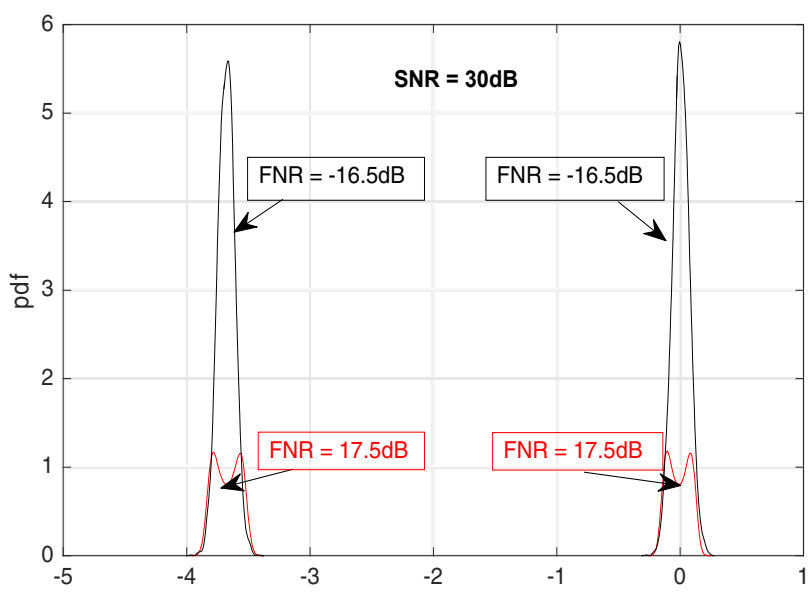

Figure 9. Pdf of the currents in the Park transformed frame

Looking at figure 9, it's clear that for incipient faults $(\mathrm{FNR}=-16.5 \mathrm{~dB})$, the pdf of the components $i_{d}$ (on the right side of the figure) and $i_{q}$ (on the left side of the figure) are close to Gaussian distributed functions. This is particularly interesting because in this case, we have a closed form of the divergence and therefore the fault characteristics can be retrieved from the divergence value. But when the fault level increases $(F N R=17.5 \mathrm{~dB})$, the pdf are distorted. In this case, the data variance, skewness or Kurtosis can be used as fault detection and estimation features. 
However, the divergence can be numerically computed from the estimated distributions and a model derived to retrieve the fault characteristics.

\section{APPLICATION TO CURRENT SENSOR FAULT ESTIMATION}

An offset $\delta I=0.6 \mathrm{~A}$ inserted (in the faulty zone) in the current measured in the phase C, representing $20 \%$ of the current amplitude at $500 \mathrm{rpm}$.

The SNR is equal to $13.3 \mathrm{~dB}$ in healthy case and $12 \mathrm{~dB}$ in the faulty case. The FNR is equal to $7 \mathrm{~dB}$.

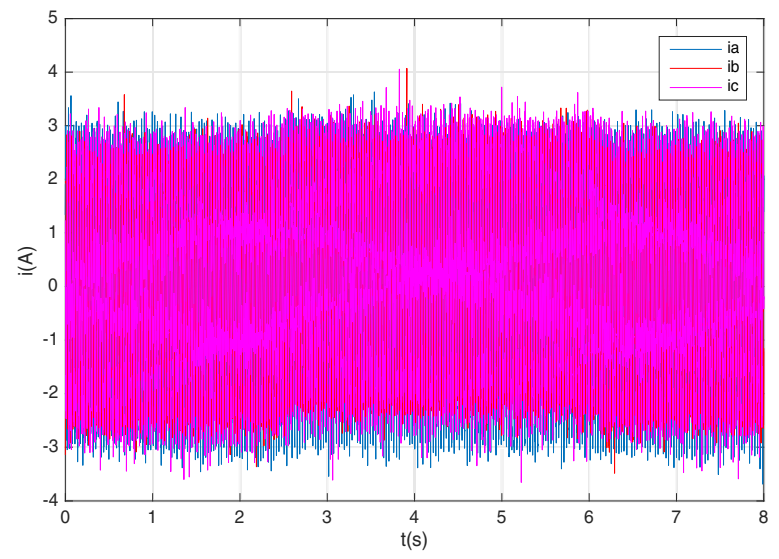

Figure 10. Transformed currents waveforms

From the waveforms of the transformed currents plotted in figure 11 , it can be observed that the main variation is observed in the $\mathrm{q}$ axis current component and that the variance has significantly changed.

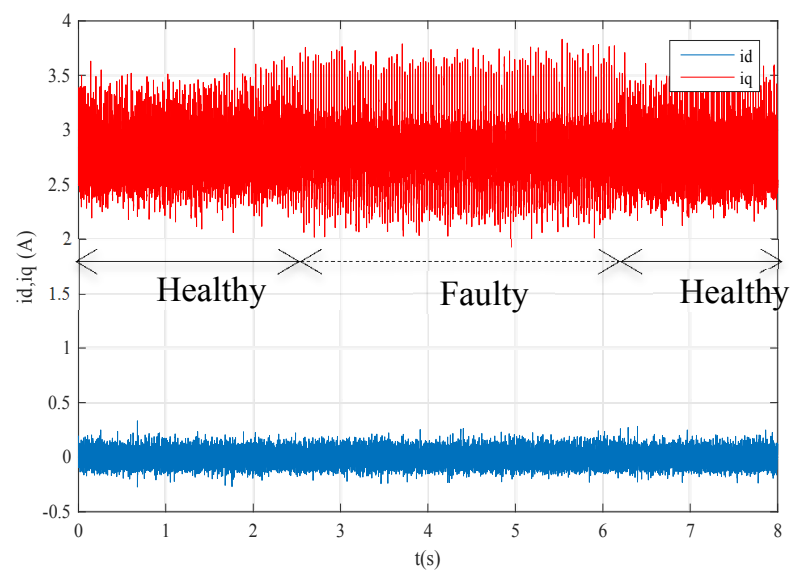

Figure 11. Transformed currents waveforms

Considering the transformed current $i_{q}$, for the healthy condition, from the measured data, the variances are $\sigma_{1}^{2}=0.0684 \sigma_{2}^{2}=0.0932$

respectively for the healthy and faulty condition.

The probability density functions of the transformed current $i_{q}$ are plotted in figure 12 for healthy and faulty conditions. The KLD can be evaluated as the pdf share the same support.

Using (7), the KLD is computed and is equal to 0.1481 .

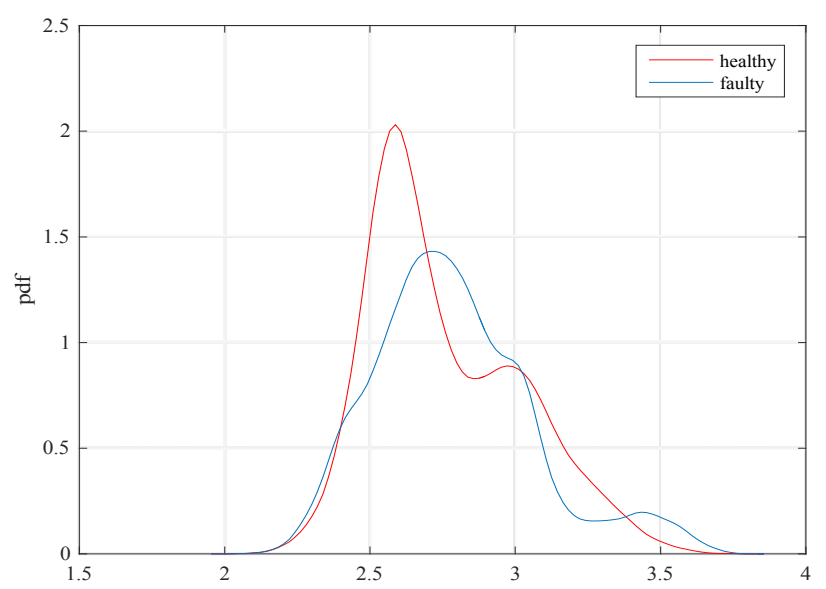

Figure 12. Pdfs of the transformed current $i_{q}$

Despite the fact that the distributions are not perfectly gaussian, (8) can be still used to retrieve the fault characteristics from the divergence.

Under the assumption that for continuous health monitoring, the healthy data (mean value and data variance $\sigma_{1}^{2}$ ) are known and the mean values before and after the fault occurrence are identical, the variance $\sigma_{2}^{2}$ in the faulty zone can be determined after the calculation of the KLD between the two pdfs and the resolution of the following polynomial derived from (8):

$$
\hat{\sigma}_{2}^{4}-(2 K L D+2) \sigma_{1}^{2} \hat{\sigma}_{2}^{2}+\sigma_{1}^{4}=0
$$

where

From the two solutions, $\begin{aligned} & \hat{\sigma}_{2,1}^{2}=0.0142, \\ & \hat{\sigma}_{2,2}^{2}=0.1741\end{aligned}$ the second solution is retained because the variance increases at fault occurrence.

As the variance $\sigma_{2}^{2}$ is related to the fault amplitude from (5), it can be deduced that:

$\sigma_{2}^{2}=\frac{(\delta I)^{2}}{3}$

As a consequence $\hat{\delta} I=3 \hat{\sigma}_{2}=0.72 \mathrm{~A}$ is the estimated offset. If we recall the initial offset, which is $0.6 \mathrm{~A}$, the estimation error is equal to $20 \%$.

This overestimation, that is rather positive for health monitoring issue, is mainly due to the assumption that the distributions are Gaussian.

Indeed this assumption is more valid for higher SNR values. For the case under study, the data are more $\Gamma$ distributed. However despite its complexity, an analytical 
model can be derived from which the fault characteristics will be extracted [13].

\section{CONCLUSION}

In this paper, a current sensor fault (bias or gain) estimation using the transformed currents in the Park synchronous rotating frame has been proposed. It has been shown that from the fault characteristics can be retrieved. From experimental raw data, the fault characteristics have been estimated with an average error of $10 \%$. But for more severe conditions (fault information concealed by the noise), despite the analytical model, the estimation might be tedious.

In this case the Kullback-Leibler Divergence between two pdfs can be computed and the fault characteristics estimated from this divergence. If the distributions are Gaussian, the closed form of the divergence allows the fault estimation.

However even if the data are not perfectly Gaussiandistributed, the closed form can still be used despite an overestimation of the fault characteristics that is preferable as it is a safety margin.

\section{REFERENCES}

1. D. Diallo, M.E.H Benbouzid, M.A Masrur, "Special Section on Condition Monitoring and Fault Accommodation in Electric and Hybrid Propulsion Systems," IEEE Transactions on Vehicular Technology, vol.62, no.3, pp.962-964, March 2013, doi: 10.1109/TVT.2013.224573

2. Campos-Delgado, D. U., Espinoza-Trejo, D. R., Palacios, E. "Fault-tolerant control in variable speed drives: a survey". IET, Electric Power Applications, vol.2, no.2, pp.121-134, 2008

3. F. Auger, M. Hilairet, J. M. Guerrero, E. Monmasson, T. Orlowska-Kowalska and S. Katsura, "Industrial Applications of the Kalman Filter: A Review," in IEEE Transactions on Industrial Electronics, vol. 60, no. 12, pp. 5458-5471, Dec. 2013.doi: 10.1109/TIE.2012.2236994

4. Rothenhagen, K., F. Fuchs W. "Current Sensor Fault Detection, Isolation, and Reconfiguration for Doubly Fed Induction Generators“ IEEE Transactions on Industrial Electronics, vol.56, no.10, pp.4239-4245, 2009

5. Lee B., Jeon N., Lee H. "Current sensor fault detection and isolation of the driving motor for an in-wheel motor drive vehicle". International conference on Control, Automation and Systems (ICCAS), vol., no., pp.486, 491, 26-29 Oct 2011

6. S. Diao, D. Diallo, C. Marchand; “An observer-based for Sensors (Position/Speed, Currents and DC-link Voltage) Fault Detection and Diagnosis in PMSM drive“, European Journal of Electrical

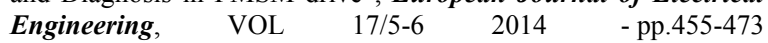
doi:10.3166/ejee.17.455-473

7. Najafabadi T., Salmasi F., Jabehdar-Maralani P. "Detection and Isolation of Speed-, DC-Link Voltage and Current-Sensor Faults Based on an Adaptive Observer in Induction-Motor Drives“, IEEE Transactions on Industrial Electronics, vol. 58, no. 5, 2011.

8. Nuno M. A. Freire, Estima J., Cardoso A. J. M. "New Approach for Current Sensor Fault Diagnosis in PMSG Drives for Wind Energy Conversion Systems“", IEEE Transactions on Industry Applications, pp. 1206-1214, 2014.

9. V. Choqueuse; A. Belouchrani; E. El Bouchiki; M. El Hachemi Benbouzid, "Estimation of Amplitude, Phase and Unbalance Parameters in Three-phase Systems: Analytical Solutions, Efficient Implementation and Performance Analysis ", IEEE Transactions on Signal Processing, Year: 2014, Volume: 62, Issue: 16 Pages: 4064 - 4076, DOI: 10.1109/TSP.2014.2333565

10. J. Harmouche, C. Delpha, D. Diallo; "Incipient Fault Detection and Diagnosis Based on Kullback - Leibler Divergence Using Principal
Component Analysis: Part I, " Signal Processing, Elsevier, Vol. 94, January 2014, pp. 278-287

11. J. Harmouche, C. Delpha, D. Diallo; "Incipient Fault Detection and Diagnosis Based on Kullback - Leibler Divergence Using Principal Component Analysis: Part II, " Signal Processing, Elsevier, April 2015, vol. 109, pp.334-344

12. M. Basseville, V. I. Nikiforov; Detection of Abrupt Changes Theory and Application, published by Prentice-Hall, Inc. (ISBN 013-126780-9 - April 1993 - Englewood Cliffs, N.J.)

13. A. Youssef, C. Delpha, and D. Diallo; "Analytical model of the KL Divergence for Gamma distributed data: application to fault estimation, “ EUSIPCO 2015, Conference Proceedings, 1-5 Sept., Nice, France 